\title{
Long-term efficacy and cost-effectiveness of blended cognitive behavior therapy for high fear of recurrence in breast, prostate and colorectal Cancer survivors: follow-up of the SWORD randomized controlled trial
}

Rens Burm ${ }^{1,2^{*}}$ (D), Belinda Thewes ${ }^{1}$, Laura Rodwell ${ }^{3}$, Wietske Kievit ${ }^{3}$, Anne Speckens ${ }^{4}$, Marieke van de Wal ${ }^{1,5}$ and Judith Prins ${ }^{1}$

\begin{abstract}
Background: Blended cognitive behaviour therapy (bCBT) is an effective treatment for fear of cancer recurrence $(F C R)$ in curatively-treated breast, colorectal and prostate cancer survivors with high FCR. However, long-term outcomes are unknown. This study investigated the long-term efficacy and cost-effectiveness of bCBT compared with care as usual (CAU).

Methods: Eighty-eight cancer survivors with high FCR (Cancer Worry Scale $\geq 14$ ) were randomly assigned to bCBT $(n=45)$ or CAU $(n=43)$. Data were collected at baseline and at three, nine and fifteen months from baseline and analysed by modified intention-to-treat. Efficacy was investigated with linear mixed-effects models. Costeffectiveness was investigated from a societal perspective by comparing costs with quality-adjusted life-years (QALYS).
\end{abstract}

Results: Participants who received bCBT reported significantly lower FCR compared with CAU (mean difference of -1.787 [95\% Cl -3.251 to $-0.323, p=0.017]$ at 15 months follow-up), and proportionally greater self-rated and clinically significant improvement at each follow-up measurement. Total QALYs were non-significantly different between conditions when adjusted for utility score baseline differences (0.984 compared to 0.957, $p=0.385$ ), while total costs were $€ 631$ lower ( $95 \% \mathrm{Cl}-1737$ to $2794, p=0.587$ ). Intervention costs of bCBT were $€ 466$. The incremental cost-effectiveness ratio amounted to an additional €2049 per QALY gained, with a 62\% probability that bCBT is cost-effective at a willingness to pay (WTP) threshold of $€ 20,000$ per QALY. Results were confirmed in sensitivity analyses.

Conclusions: bCBT for cancer survivors with FCR is clinically and statistically more effective than CAU on the long-term. In addition, bCBT is a relatively inexpensive intervention with similar costs and QALYS as CAU.

Trial registration: The RCT was registered in the Dutch National Trial Register (NTR4423) on 12-Feb-2014. This abstract was previously presented at the International Psycho-Oncology Society conference of 2018 and published online. (Psycho-oncology, 27(S3):8-55; 2018)

Keywords: Psycho-oncology, Survivorship, Fear of cancer recurrence, Blended cognitive behaviour therapy, Cost-effectiveness, Randomized controlled trial

\footnotetext{
* Correspondence: rens.burm@radboudumc.nl

'Department of Medical Psychology, Radboud university medical center,

Radboud Institute for Health Sciences, Nijmegen, The Netherlands

${ }^{2}$ MedValue, PO Box 9101, 6500, HB, Nijmegen, The Netherlands

Full list of author information is available at the end of the article
}

(c) The Author(s). 2019 Open Access This article is distributed under the terms of the Creative Commons Attribution 4.0 International License (http://creativecommons.org/licenses/by/4.0/), which permits unrestricted use, distribution, and reproduction in any medium, provided you give appropriate credit to the original author(s) and the source, provide a link to the Creative Commons license, and indicate if changes were made. The Creative Commons Public Domain Dedication waiver (http://creativecommons.org/publicdomain/zero/1.0/) applies to the data made available in this article, unless otherwise stated. 


\section{Background}

Fear of cancer recurrence (FCR), the "fear, worry, or concern relating to the possibility that cancer will come back or progress" [1, 2], is highly prevalent amongst cancer survivors. Although some degree of FCR is normal and adaptive, high levels are associated with reduced quality of life, functional impairment, distress, anxiety, depression, excessive reassurance-seeking and increased healthcare costs [3-8]. It is estimated that around a third (31-38\%) of Dutch curatively-treated cancer survivors experience high FCR [3, 9-11]. FCR seems to be a universal concern of cancer survivors rather than a cancer type-specific problem, estimates of FCR did not differ by cancer types [12]. There is consistent evidence that younger age, presence and severity of physical symptoms, psychological distress, lower quality of life, female gender and lower education are associated with higher FCR [3].

Four randomised controlled trials (RCTs) of therapist-delivered interventions to help cancer survivors manage FCR have been published to date [13-16]. Although the theoretical foundations, formats and delivery methods of these interventions differ, all available treatments are based on cognitive behaviour therapy (CBT) and some are delivered online via the internet (eHealth) [14, 17, 18]. Online CBT is a promising and potentially cost-effective treatment modality [19-21], which is at least as effective as face-to-face CBT for many mental disorders [22-25]. Online CBT may reduce waiting lists, travel time and costs [26], productivity losses [20, 26], and improve accessibility [20]. Possible disadvantages of online therapy include a lack of personal interaction, poorer adherence and less engagement [19]. Blended therapy, in which face-to-face and online therapy are combined, might bridge this gap. The Survivors' Worries of Recurrent Disease (SWORD) study is a blended CBT (bCBT) treatment for high FCR. Immediate post-treatment efficacy has been established in an RCT which compared bCBT with care as usual (CAU) in Dutch cancer survivors with high FCR [14]. FCR was significantly reduced at 3 months follow-up in participants who received bCBT compared to those who received CAU (Cancer Worry Scale mean difference of $-3.48,95 \%$ CI -4.69 to $-2.28, p<0.001$ ) with a medium to large effect size $(d=0.76)$ [14]. More detailed methodology and results are reported elsewhere [14, 27]. Efficacy at 6 and 11 months post-treatment has been demonstrated for two other FCR interventions [13, 16], however efficacy beyond the first year after treatment has received little attention. Only one trial to date has included a follow-up beyond the first year after treatment ended; this study of a therapist-delivered FCR intervention found that treatment was not more effective than CAU in reducing FCR at 15 months [15]. No previous studies have evaluated the cost-effectiveness of an individual psychological intervention for FCR, however one study has evaluated the cost-effectiveness of two group-based interventions to reduce fear of progression in cancer patients of mixed disease stage [28]. This study found that group CBT was less costly than supportive-experiential group therapy with a similar reduction of fear of progression [28].

With increasingly constrained healthcare budgets it is important to ensure that the benefits of an intervention outweigh the costs [29]. Therefore, this study aimed to evaluate the efficacy and cost-effectiveness of the SWORD intervention at 9 and 15 months follow-up.

\section{Methods}

\section{Study design and participants}

Detailed information concerning study design, recruitment and inclusion/exclusion criteria is published elsewhere [27]. Between 2014 and 2016, a multicentre prospective two-arm RCT was conducted investigating the efficacy of bCBT in cancer survivors with high FCR (Cancer Worry Scale score $\geq 14$ ). In total, 88 curatively-treated breast (BC), prostate (PC) and colorectal cancer (CRC) survivors who had completed primary medical treatment at least six months and no longer than five years were included. Eligible participants who gave informed consent were randomly assigned to receive either CAU or bCBT. The RCT was approved by an ethical board (CMO Arnhem-Nijmegen), registered in the Dutch National Trial Register (NTR4423) and adheres to CONSORT guidelines.

\section{Intervention and care as usual}

The intervention was delivered as blended care during a 3-month period: five individual one-hour face-to-face sessions (sessions 1, 2, 3, 5, 8) combined with three 15-min e-consultations (chat application, sessions 4, 6, 7) and access to a website. Therapeutic techniques applied in the intervention included psycho-education, cognitive restructuring, and behavioral modification. One face-to-face booster session followed at three months post-therapy. Participants who received CAU had no restrictions regarding the use of other psychosocial support during the study period.

The website was developed in agreement with Dutch privacy laws equal to HIPAA. All communication from and to their servers was encrypted. The hosting network was certified.

\section{Outcome measures}

Questionnaires were completed before randomization (baseline, T0), and at three months (T1), nine months (T2) and fifteen months (T3) after baseline assessment. The primary outcome measure was FCR, assessed with 
the Cancer Worry Scale (CWS). The CWS is a reliable and valid questionnaire which measures FCR on an 8 -item 4-level scale [10]. Total scores range between 8 and 32 and a cut-off score of $\leq 13$ versus $\geq 14$ differentiates between low and high FCR [10]. Secondary outcomes were FCR severity (Fear of Cancer Recurrence Inventory [FCRI] severity subscale) [30], cancer-specific distress (Impact of Events Scale [IES]) [31, 32], distress (Hospital Anxiety and Depression Scale [HADS]) [33], fatigue (Checklist Individual Strength 8R [CIS-8R]) [34], and health-related quality of life (HRQoL; European Organisation for Research and Treatment of Cancer Quality of Life Questionnaire C30 [EORTC QLQ-C30]) [35].

Costs and quality-adjusted life years (QALYs) were used as the outcome measures in the cost-effectiveness analyses. The QALY combines quality (weighed by utility scores) and quantity of life (life years gained) in one measure and is the predominant outcome measure used in health economic evaluations to assess value for money of interventions. QALYs enable comparison of cost-effectiveness of different interventions across different disease areas. Each QALY gained is worth a certain maximum monetary value that governmental organizations or insurance companies are willing to pay; also called willingness to pay (WTP) thresholds. To calculate QALYs, utility scores were obtained by using the EuroQol five dimensions three-level questionnaire (EQ-5D-3 L) and by applying the Dutch EQ-5D tariff $[36,37]$. Utility scores are preference-based quality of life values, generally ranging from 0 (death) to 1 (perfect health). These utilities were multiplied by the duration of follow-up (fifteen months) to calculate QALYs.

The economic evaluation was conducted from a societal perspective, and therefore included medical costs (healthcare resource use and medication), non-medical costs (informal care and loss of productivity from paid work) and costs of the bCBT intervention. Both medical- and non-medical resource use were measured by pre-defined self-reported cost diaries collected at T1, T2 and T3. Resource use volumes were valued using standard Dutch reference prices for 2014 if available (Additional file 1: Appendix 1) [29]. The economic impact of absence from paid work was calculated using the friction cost method [38], which was set at 12 weeks in 2014 in the Netherlands [29]. Intervention program costs included bottom-up calculated costs of psychologist training, supervision and consultations, in addition to costs of website development, updates and user licences divided by a conservative amount of potential annual users in a regional implementation scenario (Additional file 1: Appendix 2) $[39,40]$. No costs or effects were discounted due to the short study period.

\section{Statistical analysis}

For the analysis of the primary outcome of FCR, all secondary outcomes and health-economic outcomes, a modified intention-to-treat (mITT) analysis was performed; in which participants who experienced a recurrence of cancer during the follow-up period were retained in the analysis, but only their scores before recurrence were included in the analysis.

\section{Clinical outcomes}

Long-term effects of bCBT on primary and secondary outcomes were examined by fitting a linear mixed-effects model for mean CWS follow-up scores. This model included treatment condition, follow-up time (using discrete time points) and their interaction, with an unstructured residual-error covariance matrix specified to account for the within-person correlation on the repeated follow-up measures. Baseline score and primary cancer type were included as covariates. The latter was a stratification variable for randomisation and was also found to be associated with the probability of missing data at T2 and T3. For the primary outcome of FCR, two sensitivity analyses were conducted. In the first sensitivity analysis, participants who reported a recurrence were excluded from the analysis dataset. The second sensitivity analysis was a per protocol analysis of participants who completed the bCBT intervention (intervention arm) and who had full follow-up data on FCR. All analyses for the clinical outcomes were conducted in Stata 14.2.

Clinically significant improvement (CSI) on levels of FCR between baseline and both T2 and T3 follow-up assessments was established by combining statistically reliable improvement (reliable change index < 1.96) [41] and a decrease of CWS scores to the normal range (clinically significant change, CWS < 14) [27]. Self-rated improvement had occurred if the participant answered 'yes' to 'feeling much better but still experiencing some FCR' or 'no longer experiencing FCR'.

\section{Health economic outcomes}

Missing total cost and utility score data per measurement were imputed at the aggregated level separately per treatment arm, by using multiple imputation (MI) by chained equations with predictive mean-matching $[42,43]$. Twenty imputed datasets were created and pooled according to Rubin's rules [44].

First of all, incremental costs and incremental QALYs between the CAU and bCBT strategies were calculated and statistical differences in non-normally distributed costs and QALYs were tested with bootstrapped $\mathrm{t}$-tests [45]. Non-parametric percentile bootstrapping with 1000 replications was performed to estimate $95 \%$ confidence intervals [46]. In order to weigh incremental costs against incremental QALYs, the incremental cost-effectiveness 
ratio (ICER) was calculated by dividing total incremental costs by the incremental QALYs to obtain the costs per QALY gained by the intervention [39]. Due to substantial differences in EQ-5D baseline utility scores between conditions, regression-based adjustment was performed within the net monetary benefit framework, which values both benefits and costs in monetary terms and therefore enables the use of regression methods [47]. Incremental net monetary benefit (INMB) statistics were estimated for different WTP thresholds. The INMB was calculated by using the following formula: (incremental QALY * WTP) - incremental costs. Cost-effectiveness acceptability curves (CEACs) were constructed which display the probability that bCBT would be cost-effective given specific WTP thresholds [48]. A sensitivity analysis was conducted which used condition-specific utility scores calculated from the EORTC QLQ-C30 instead of utility scores calculated from the EQ-5D-3 L [49].

\section{Results}

\section{Sample characteristics}

In total, 45 participants were randomly assigned to bCBT and 43 to CAU (Fig. 1). Both conditions were comparable on demographic characteristics and clinical characteristics (Table 1). Nine participants reported a recurrence at some point during follow-up (bCBT: 8, CAU: 1) and one participant receiving CAU reported a new primary cancer. Three of these patients dropped out of the study (bCBT: 2, CAU: 1). Furthermore, 16 participants dropped out of the study during follow-up for various other reasons (bCBT: 5, CAU: 11; Fig. 1). Having had colorectal cancer $(p=0.047)$ and having comorbid conditions $(p=0.044)$ was significantly associated with dropout at any stage during the study. The number of completers (who completed all CWS questionnaires and, for bCBT, the full intervention) was 23 in the $\mathrm{bCBT}$ and 30 in the CAU condition.

\section{Clinical outcomes}

Results for the primary and secondary clinical outcomes are presented in Table 2. The decrease in FCR was significantly greater in participants who received bCBT compared with CAU at all follow-up measurements (Additional file 1: Appendix 3). At T3, the detected mean difference in FCR remained statistically significant $(-1.787,95 \%$ CI -3.251 to $-0.323, p=0.017)$, although smaller than at T1 $(-3.534,95 \%$ CI -4.764 to -2.305 , $p=0.000)$ and T2 $(-4.104,95 \%$ CI -5.531 to -2.677 , $p=0.000)$. Similar results were found in the per protocol sensitivity analysis. In the sensitivity analysis which excluded participants with recurrences, the reduction in FCR in the $\mathrm{bCBT}$ condition was larger at each follow-up measurement than in the primary analysis (Additional file 1: Appendix 4).

Regarding the secondary outcomes, participants receiving bCBT experienced significantly greater improvement at each follow-up measurement on anxiety, emotional functioning and the FCRI severity subscale compared with CAU. Significant improvement at T1 and T2 was found on distress, depression and global quality of life. On the other six secondary outcomes (cancer-specific distress, fatigue and physical, role, social and cognitive functioning), a statistically significant effect was found at $\mathrm{T} 1$.

Significantly more participants receiving bCBT compared with CAU reported reliable improvement ( $78 \%$ vs. $19 \%, p \leq 0.001)$, clinically significant change (58\% vs. $9 \%, p \leq 0.001$ ), clinically significant improvement $(51 \%$ vs. $3 \%, p \leq 0.001)$ and self-rated improvement ( $74 \%$ vs. $41 \%, p=0.007)$ at T2. At T3, only clinically significant change $(50 \%$ vs. $16 \%, p=0.038)$ and self-rated improvement ( $72 \%$ vs. $40 \%, p=0.020)$ remained significantly higher in bCBT (Additional file 1: Appendix 5).

\section{Health economic outcomes}

Participants receiving bCBT reported substantially lower costs on most medical resource categories, including medical specialists, psychosocial help and diagnostics (Additional file 1: Appendix 6). Costs of the bCBT intervention programme amounted to $€ 466$ (Additional file 1: Appendix 2), which were added to the costs made by bCBT participants. Total costs (combined medical, non-medical and bCBT intervention costs) over the complete follow-up period were non-significantly lower in those who received bCBT compared with CAU ( $-€ 164,95 \%$ CI -2018 to $2502, p=0.891$ ).

Results of the different cost-effectiveness analyses are shown in Table 3. Baseline EQ-5D utility scores were substantially higher in the bCBT condition $(0.78[\mathrm{SD}=0.18])$ than in CAU (0.68 [SD = 0.22]) (Additional file 1: Appendix 3). Mean QALYs unadjusted for this baseline difference were significantly higher in the bCBT condition (1.027 compared to $0.913, p=0.018$ ). However, QALYs were non-significantly different when these were adjusted for baseline EQ-5D utility scores (0.984 compared to $0.957, p$ $=0.385)$ and when QALYs were calculated from QLQ-C30 utility scores (1.107 compared to $1.074, p=0.137$ ).

Since the total costs were lower and total QALYs were higher when QALYs were unadjusted for baseline utility differences, bCBT was the dominant strategy (negative ICER) in this analysis. The same applies to the analysis which used QALYs calculated from QLQ-C30 utility scores instead of EQ-5D utility scores. An ICER of $€ 2049$ per QALY gained was found when QALYs were adjusted for baseline EQ-5D utility differences, since bCBT was both less costly and less effective in this 


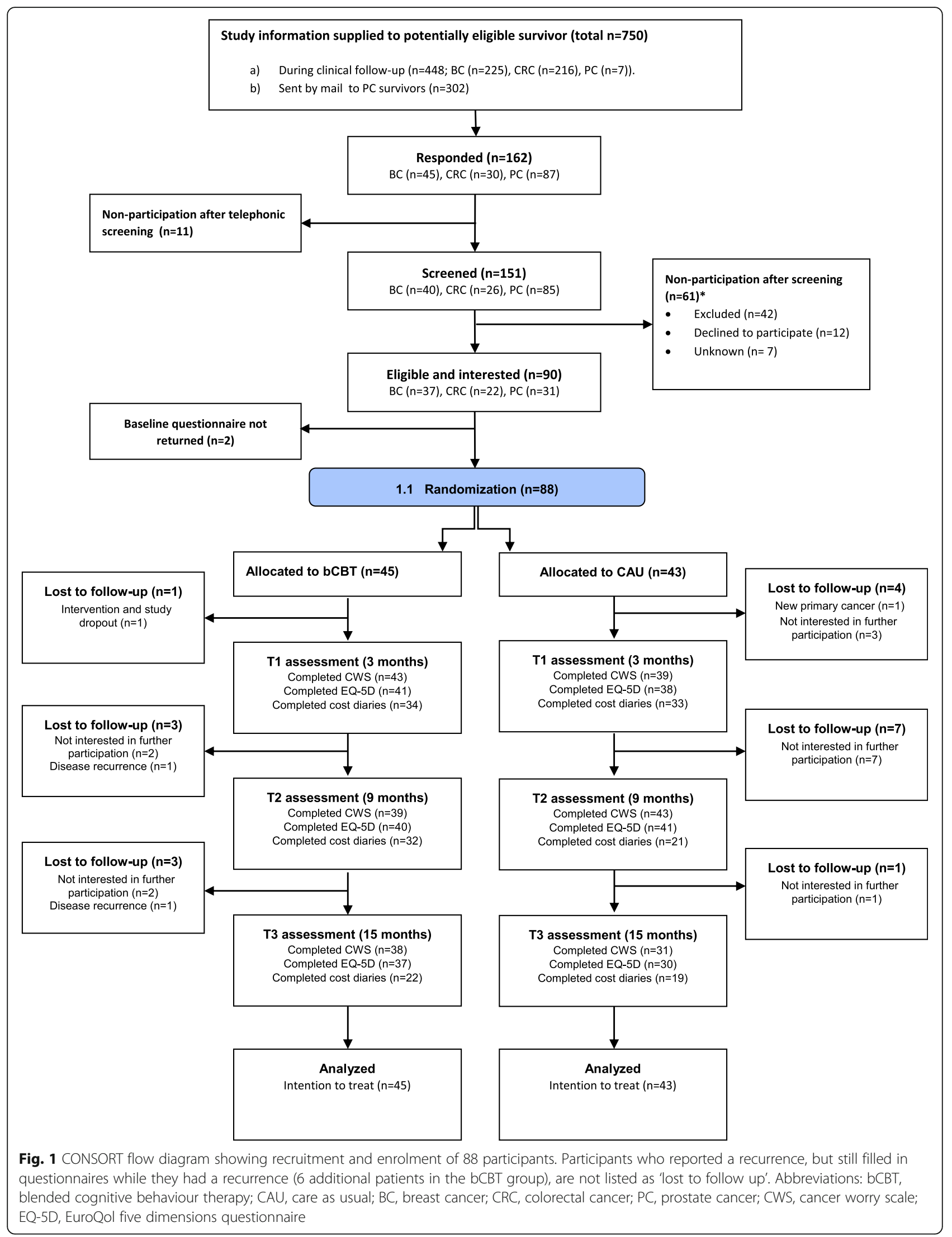


Table 1 Demographic and clinical characteristics at baseline of participants by study group

\begin{tabular}{|c|c|c|}
\hline & $\begin{array}{l}\text { bCBT } \\
(n=45)\end{array}$ & $\begin{array}{l}\text { CAU } \\
(n=43)\end{array}$ \\
\hline Characteristic & $n(\%)$ & $n(\%)$ \\
\hline \multicolumn{3}{|l|}{ Age } \\
\hline Mean (SD) & $58.0(11.3)$ & $59.7(10.0)$ \\
\hline \multicolumn{3}{|l|}{ Gender } \\
\hline Male & $21(46.7 \%)$ & $20(46.5 \%)$ \\
\hline \multicolumn{3}{|l|}{ Education } \\
\hline Low (ISCED 0-2) & $11(24.4 \%)$ & $18(41.9 \%)$ \\
\hline Middle (ISCED 3-5) & 19 (42.2\%) & $10(23.3 \%)$ \\
\hline High (ISCED 6-8) & $15(33.3 \%)$ & $15(34.9 \%)$ \\
\hline \multicolumn{3}{|l|}{ Employment status } \\
\hline Paid Employment & 17 (37.8\%) & 17 (39.5\%) \\
\hline Unemployed & $8(17.8 \%)$ & $5(11.6 \%)$ \\
\hline Voluntary work & $4(8.9 \%)$ & $8(18.6 \%)$ \\
\hline Retired & $16(35.6 \%)$ & $14(32.6 \%)$ \\
\hline \multicolumn{3}{|l|}{ Primary cancer site } \\
\hline Breast & $18(40.0 \%)$ & $18(41.9 \%)$ \\
\hline Colorectal & $12(26.7 \%)$ & $10(23.3 \%)$ \\
\hline Prostate & 15 (33.3\%) & $15(34.9 \%)$ \\
\hline \multicolumn{3}{|l|}{ Treatment type } \\
\hline Surgery & $18(40.0 \%)$ & $11(25.6 \%)$ \\
\hline Radiotherapy & $0(0.0 \%)$ & $3(7.0 \%)$ \\
\hline Surgery + Radiotherapy & $9(20.0 \%)$ & 9 (20.9\%) \\
\hline Surgery + Chemotherapy & $12(26.7 \%)$ & $9(20.9 \%)$ \\
\hline Surgery + Radiotherapy + Chemotherapy & $6(13.3 \%)$ & $11(25.6 \%)$ \\
\hline \multicolumn{3}{|l|}{ Time since diagnosis (years) } \\
\hline Mean (SD) & $2.4(1.5)$ & $2.8(1.3)$ \\
\hline \multicolumn{3}{|l|}{ Time since last treatment (years) } \\
\hline Mean (SD) & $1.9(1.5)$ & $2.1(1.4)$ \\
\hline \multicolumn{3}{|l|}{ Number of comorbid diseases } \\
\hline 0 & $19(42.2 \%)$ & $14(32.6 \%)$ \\
\hline $1-2$ & $19(42.2 \%)$ & $22(51.2 \%)$ \\
\hline $3+$ & $7(15.6 \%)$ & $7(16.3 \%)$ \\
\hline \multicolumn{3}{|l|}{ Psychological help in the past } \\
\hline yes & $22(50.0 \%)$ & $26(60.5 \%)$ \\
\hline
\end{tabular}

Abbreviations: $b C B T$ blended cognitive behaviour therapy, $C A U$ care as usual, ISCED International Standard Classification of Education, SD standard deviation

analysis. INMBs in the unadjusted and QLQ-C30 analyses were in favour of the bCBT condition regardless of society's WTP per QALY gained, while adjusted INMBs were in favour of bCBT above a WTP of $€ 2049$ per QALY gained (which is equal to the ICER). However, all INMBs had wide confidence intervals, most of which included 0 at all WTP values. The CEAC shows that there is a 48 to $56 \%$ probability that bCBT is cost-saving compared to $\mathrm{CAU}$, and a 62 to $95 \%$ probability that bCBT is more cost-effective than CAU at a WTP of $€ 20,000$ (Fig. 2). This probability obviously increases as WTP values increase.

\section{Discussion}

This study investigated the long-term efficacy and cost-effectiveness of bCBT to reduce FCR in PC, BC and CRC survivors with high FCR. It was shown previously that bCBT resulted in a statistically and clinically significant reduction of FCR severity immediately posttreatment (3 months) compared with CAU [14]. The current study found that the effect of bCBT remained significant during follow-up at 9 and 15 months [50]. Findings were confirmed in the sensitivity analyses. Furthermore, more bCBT than CAU participants reported self-rated improvement and clinically significant improvement at 9 and 15 months. However, the difference in clinically significant improvement at T3 was not statistically significant, reflecting the finding that FCR increased slightly in the bCBT condition and significantly decreased in the CAU condition between 9 and 15 months from baseline. The decrease in FCR over time in the CAU condition is inconsistent with previous literature reviews stating that high FCR remains stable over time without intervention $[3,5,51,52]$. However, CAU participants received considerably more additional psychosocial support (by either a psychologist, mental health nurse, social worker or psychiatrist) than bCBT participants which may explain this decrease. For secondary outcomes, those receiving bCBT improved significantly on multidimensional aspects of FCR, anxiety and emotional functioning at each follow-up measurement. Other secondary outcomes (distress, depression, global quality of life) improved at $\mathrm{T} 1$ and $\mathrm{T} 2$, while the remainder (cancer-specific distress, fatigue and physical, role, social and cognitive functioning) only improved at 3 months follow-up.

In the economic evaluation, total costs were found to be numerically but not significantly lower in the bCBT condition. Intervention costs were relatively low when calculated for a conservative annual number of potential users. The amount of additional QALYs was small and not statistically significant when adjusted for baseline EQ-5D utility scores and in the sensitivity analysis using QLQ-C30 utility scores. All cost-effectiveness analyses resulted in low costs of bCBT per QALY gained and relatively high probabilities that bCBT is cost-saving compared to CAU. The maximum WTP for an additional QALY in the Netherlands is dependent on the burden of disease of the addressed condition, ranging from $€ 20,000$ to $€ 80,000$ [53]. As the disease burden of high FCR is not yet reported, we decided to use the 
Table 2 Effect of treatment (modified intention-to-treat) on primary and secondary outcomes $(n=88)$

\begin{tabular}{|c|c|c|c|c|c|c|c|}
\hline & \multicolumn{2}{|c|}{$\mathrm{bCBT}(n=45)$} & \multicolumn{2}{|c|}{$\mathrm{CAU}(n=43)$} & \multirow[b]{2}{*}{ Mean difference } & \multirow[b]{2}{*}{$95 \% \mathrm{Cl}$} & \multirow[b]{2}{*}{$p$ value } \\
\hline & Mean & $\mathrm{SE} / \mathrm{SD}^{*}$ & Mean & $\mathrm{SE} / \mathrm{SD}^{*}$ & & & \\
\hline \multicolumn{8}{|l|}{ Primary outcome } \\
\hline \multicolumn{8}{|l|}{ FCR (CWS) } \\
\hline TO & 19.622 & 3.737 & 19.558 & 3.744 & & & \\
\hline T1 unadjusted & 14.814 & 4.579 & 18.487 & 3.986 & & & \\
\hline T1 adjusted & 14.743 & 0.432 & 18.277 & 0.454 & -3.534 & -4.764 to -2.305 & 0.000 \\
\hline T2 unadjusted & 13.718 & 4.472 & 17.844 & 3.836 & & & \\
\hline T2 adjusted & 13.916 & 0.493 & 18.020 & 0.534 & -4.104 & -5.531 to -2.677 & 0.000 \\
\hline T3 unadjusted & 14.684 & 4.539 & 16.516 & 4.081 & & & \\
\hline T3 adjusted & 14.783 & 0.504 & 16.570 & 0.549 & -1.787 & -3.251 to -0.323 & 0.017 \\
\hline
\end{tabular}

Secondary outcomes

Multidimensional aspects of FCR

Severity (FCRI)

$\begin{array}{lllll}\text { T0 } & 21.909 & 4.850 & 23.116 & 6.45 \\ \text { T1 unadjusted } & 16.605 & 7.251 & 22.282 & 5.99 \\ \text { T1 adjusted } & 16.989 & 0.756 & 21.330 & 0.788 \\ \text { T2 unadjusted } & 15.025 & 6.897 & 21.313 & 5.17 \\ \text { T2 adjusted } & 15.957 & 0.688 & 20.462 & 0.74 \\ \text { T3 unadjusted } & 15.842 & 7.343 & 19.733 & 5.53 \\ \text { T3 adjusted } & 16.599 & 0.805 & 19.023 & 0.879\end{array}$

6.456

5.991

0.788

5.171

0.742

5.539

0.879

$-4.341$

-6.497 to -2.185

0.000

ss, depression, anxiety

Distress (HADS)

TO

T1 unadjusted

14.089

7.836

15.209

8.911

T1 adjusted

9.951

8.402

17.131

9.519

10.749

0.731

T2 unadjusted

9.718

7.412

T2 adjusted

11.139

0.806

9.778

8.292

T3 adjusted

11.414

0.966

Anxiety (HADS)

T0

T1 unadjusted

8.156

4.101

T1 adjusted

5.317

4.239

T2 unadjusted

5.639

0.437

4.132

T2 adjusted

5.333

6.030

0.528

4.944

4.056

0.570

T3 adjusted

5.751

Depression (HADS)

TO

T1 unadjusted

5.933

4.218

T1 adjusted

4.634

4.598

0.424

T2 unadjusted

5.050

4.017

0.418
5.042
16.003

0.763

15.862

9.771

14.897

0.898

14.889

9.390

13.228

1.082

8.395

4.914

9.447

8.951

8.862

8.549

8.333

7.734

6.814

4.722

7.684

7.131

7.000
5.012

0.456

5.585

0.588

4.844

0.638

5.152

0.442

4.751

0.467

$$
-5.254
$$$$
-7.334 \text { to }-3.174
$$$$
-3.759
$$$$
-1.813
$$$$
-6.134 \text { to }-1.383
$$$$
-4.669 \text { to } 1.043
$$

$-3.312$

$-2.519$

$-1.984$

-3.667 to -0.301

0.021

6.406

$\begin{array}{ll}-2.081 & -3.286 \text { to }-0.876 \\ -1.363 & -2.597 \text { to }-0.130\end{array}$

0.001

0.030 
Table 2 Effect of treatment (modified intention-to-treat) on primary and secondary outcomes $(n=88)$ (Continued)

\begin{tabular}{|c|c|c|c|c|c|c|c|}
\hline & \multicolumn{2}{|c|}{$\mathrm{bCBT}(n=45)$} & \multicolumn{2}{|c|}{$\mathrm{CAU}(n=43)$} & \multirow[b]{2}{*}{ Mean difference } & \multirow[b]{2}{*}{$95 \% \mathrm{Cl}$} & \multirow[b]{2}{*}{$p$ value } \\
\hline & Mean & $\mathrm{SE} / \mathrm{SD}^{*}$ & Mean & SE/SD* & & & \\
\hline T3 unadjusted & 4.833 & 4.675 & 6.556 & 4.956 & & & \\
\hline T3 adjusted & 5.582 & 0.504 & 5.611 & 0.565 & -.029 & -1.520 to 1.462 & 0.970 \\
\hline \multicolumn{8}{|c|}{ Cancer-specific distress (IES) } \\
\hline T0 & 23.044 & 14.555 & 24.902 & 15.604 & & & \\
\hline T1 unadjusted & 14.732 & 14.998 & 26.026 & 17.747 & & & \\
\hline T1 adjusted & 15.539 & 2.064 & 24.598 & 2.208 & -9.059 & -15.003 to -3.115 & 0.003 \\
\hline T2 unadjusted & 9.973 & 13.710 & 16.643 & 15.905 & & & \\
\hline T2 adjusted & 11.127 & 2.085 & 17.107 & 2.391 & -5.979 & -12.215 to 0.256 & 0.060 \\
\hline T3 unadjusted & 14.944 & 14.633 & 18.259 & 15.294 & & & \\
\hline T3 adjusted & 15.781 & 2.147 & 17.254 & 2.450 & -1.473 & -7.883 to 4.938 & 0.653 \\
\hline \multicolumn{8}{|l|}{ Fatigue (CIS-8R) } \\
\hline T0 & 30.222 & 12.951 & 36.116 & 12.585 & & & \\
\hline T1 unadjusted & 25.250 & 11.535 & 36.684 & 13.001 & & & \\
\hline T1 adjusted & 27.961 & 1.137 & 33.478 & 1.176 & -5.516 & -8.771 to -2.262 & 0.001 \\
\hline T2 unadjusted & 26.769 & 11.855 & 32.586 & 12.290 & & & \\
\hline T2 adjusted & 28.694 & 1.297 & 30.512 & 1.444 & -1.818 & -5.658 to 2.022 & 0.353 \\
\hline T3 unadjusted & 26.806 & 11.436 & 30.556 & 13.042 & & & \\
\hline T3 adjusted & 29.387 & 1.350 & 26.888 & 1.514 & 2.499 & -1.522 to 6.521 & 0.223 \\
\hline
\end{tabular}

Quality of life (EORTC QLQ-C30)

Global quality of life

\begin{tabular}{|c|c|c|c|c|c|c|c|}
\hline T0 & 62.222 & 19.672 & 61.434 & 20.252 & & & \\
\hline T1 unadjusted & 73.062 & 19.402 & 57.479 & 20.483 & & & \\
\hline T1 adjusted & 72.504 & 2.556 & 58.075 & 2.682 & 14.429 & 7.153 to 21.705 & 0.000 \\
\hline T2 unadjusted & 72.083 & 21.229 & 62.500 & 21.792 & & & \\
\hline T2 adjusted & 71.918 & 3.004 & 62.632 & 3.264 & 9.286 & 0.576 to 17.997 & 0.037 \\
\hline T3 unadjusted & 73.026 & 19.609 & 65.278 & 19.948 & & & \\
\hline T3 adjusted & 72.672 & 2.823 & 67.742 & 3.108 & 4.930 & -3.331 to 13.192 & 0.242 \\
\hline \multicolumn{8}{|c|}{ hysical functioning } \\
\hline TO & 87.704 & 13.406 & 83.953 & 16.269 & & & \\
\hline T1 unadjusted & 89.380 & 11.735 & 80.855 & 20.010 & & & \\
\hline T1 adjusted & 87.552 & 1.527 & 82.886 & 1.606 & 4.666 & .296 to 9.037 & 0.036 \\
\hline T2 unadjusted & 88.750 & 15.148 & 86.458 & 14.809 & & & \\
\hline T2 adjusted & 86.246 & 1.737 & 87.766 & 1.893 & -1.519 & -6.583 to 3.545 & 0.557 \\
\hline T3 unadjusted & 89.649 & 13.924 & 84.301 & 14.560 & & & \\
\hline T3 adjusted & 87.986 & 1.592 & 86.829 & 1.721 & 1.156 & -3.479 to 5.792 & 0.625 \\
\hline \multicolumn{8}{|l|}{ ole functioning } \\
\hline TO & 77.037 & 26.181 & 74.806 & 25.295 & & & \\
\hline T1 unadjusted & 85.659 & 21.076 & 74.123 & 23.474 & & & \\
\hline T1 adjusted & 84.543 & 2.963 & 75.547 & 3.142 & 8.996 & 0.503 to 17.489 & 0.038 \\
\hline T2 unadjusted & 83.750 & 23.415 & 82.292 & 21.972 & & & \\
\hline T2 adjusted & 82.766 & 3.407 & 82.705 & 3.742 & 0.061 & -9.887 to 10.008 & 0.991 \\
\hline T3 unadjusted & 89.035 & 17.013 & 84.409 & 23.148 & & & \\
\hline T3 adjusted & 87.388 & 2.777 & 85.357 & 3.043 & 2.031 & -6.095 to 10.157 & 0.624 \\
\hline
\end{tabular}


Table 2 Effect of treatment (modified intention-to-treat) on primary and secondary outcomes $(n=88)$ (Continued)

\begin{tabular}{|c|c|c|c|c|c|c|c|}
\hline & \multicolumn{2}{|c|}{$\mathrm{bCBT}(n=45)$} & \multicolumn{2}{|c|}{$\mathrm{CAU}(n=43)$} & \multirow[b]{2}{*}{ Mean difference } & \multirow[b]{2}{*}{$95 \% \mathrm{Cl}$} & \multirow[b]{2}{*}{$p$ value } \\
\hline & Mean & SE/SD* & Mean & SE/SD* & & & \\
\hline \multicolumn{8}{|c|}{ Emotional functioning } \\
\hline T0 & 64.815 & 22.883 & 66.860 & 22.456 & & & \\
\hline T1 unadjusted & 81.589 & 23.185 & 60.256 & 29.830 & & & \\
\hline T1 adjusted & 81.647 & 2.934 & 59.978 & 3.078 & 21.669 & 13.326 to 30.011 & 0.000 \\
\hline T2 unadjusted & 80.208 & 22.463 & 61.719 & 28.544 & & & \\
\hline T2 adjusted & 78.959 & 3.259 & 60.785 & 3.540 & 18.174 & 8.731 to 27.617 & 0.000 \\
\hline T3 unadjusted & 77.851 & 23.592 & 68.548 & 25.973 & & & \\
\hline T3 adjusted & 77.507 & 2.987 & 68.668 & 3.242 & 8.839 & 0.179 to 17.499 & 0.046 \\
\hline \multicolumn{8}{|c|}{ Cognitive functioning } \\
\hline T0 & 72.963 & 24.692 & 68.992 & 25.350 & & & \\
\hline T1 unadjusted & 80.620 & 19.897 & 62.393 & 28.541 & & & \\
\hline T1 adjusted & 79.977 & 2.920 & 64.662 & 3.071 & 15.315 & 6.989 to 23.641 & 0.000 \\
\hline T2 unadjusted & 82.500 & 19.954 & 72.917 & 28.945 & & & \\
\hline T2 adjusted & 80.130 & 3.276 & 74.990 & 3.593 & 5.140 & -4.421 to 14.701 & 0.292 \\
\hline T3 unadjusted & 79.386 & 22.738 & 68.280 & 25.587 & & & \\
\hline T3 adjusted & 76.994 & 3.171 & 71.780 & 3.442 & 5.214 & -4.013 to 14.441 & 0.268 \\
\hline \multicolumn{8}{|l|}{ Social functioning } \\
\hline T0 & 80.000 & 23.192 & 71.318 & 24.754 & & & \\
\hline T1 unadjusted & 89.922 & 17.495 & 76.923 & 26.384 & & & \\
\hline T1 adjusted & 87.868 & 2.907 & 79.225 & 3.055 & 8.643 & 0.306 to 16.979 & 0.042 \\
\hline T2 unadjusted & 85.833 & 22.504 & 80.208 & 22.175 & & & \\
\hline T2 adjusted & 83.302 & 3.052 & 81.127 & 3.310 & 2.176 & -6.727 to 11.078 & 0.632 \\
\hline T3 unadjusted & 88.158 & 19.723 & 86.022 & 19.292 & & & \\
\hline T3 adjusted & 85.924 & 2.556 & 89.059 & 2.759 & 3.135 & -10.641 to 4.370 & 0.413 \\
\hline
\end{tabular}

NOTE. Linear mixed-effects models were used to calculate all $p$-values. Adjusted means were adjusted for the baseline covariates primary cancer site and baseline value of the corresponding outcome; unadjusted means were unadjusted for covariates. Boldface type indicates a statistically significant effect for adjusted means $(p<0.05)$

*SEs are reported for adjusted means, SDs for unadjusted means

Abbreviations: $b C B T$ blended cognitive behaviour therapy, CAU care as usual; FCR fear of cancer recurrence, CWS Cancer Worry Scale, FCRI Fear of Cancer Recurrence Inventory, HADS Hospital Anxiety and Depression Scale, IES Impact of Events Scale, CIS-8R Checklist Individual Strength 8R, EORTC QLQ-C30 European Organisation for Research and Treatment of Cancer Quality of Life Questionnaire C30, SD standard deviation, SE standard error

most conservative WTP of $€ 20,000$; at which the probability that bCBT is cost effective ranged from 62 to 95\%. However, since total costs and QALYs were not significantly different and the INMBs had relatively wide confidence intervals which included zero, it cannot be concluded that bCBT is more cost-effective than CAU. Instead, it could be concluded that bCBT and CAU are similar in terms of costs and QALYs. Nonetheless, as costs of eHealth interventions are partly dependant on the number of users [54], it is envisaged that when implemented in routine care, the intervention will probably become more cost-effective than demonstrated here.

Like other recent RCTs of psychological interventions, the present study has demonstrated immediate and medium-term benefits of a CBT-based psychological intervention for FCR. However, to our knowledge, this is the first study to demonstrate efficacy and cost-effectiveness of a psychological intervention for FCR beyond the first year after treatment. Several on-going trials of interventions for FCR include cost-effectiveness outcomes, and it will be interesting to compare health-economic results [55-57].

Methodological strengths of this study include the rigorous $\mathrm{RCT}$ design and the broad range of different clinical and health economic outcomes. Both statistical and clinical change was evaluated, and findings were adjusted for covariates and confirmed by sensitivity analyses. Despite being adequately powered, a relatively high number of dropouts (18\%) and 
Table 3 Results of the different cost-effectiveness analyses

\begin{tabular}{|c|c|c|c|c|c|c|c|}
\hline & & \multicolumn{2}{|l|}{ Unadjusted } & \multicolumn{2}{|l|}{ Adjusted } & \multicolumn{2}{|l|}{ QLQ-C30 } \\
\hline & & bCBT & CAU & bCBT & CAU & bCBT & CAU \\
\hline \multirow[t]{2}{*}{ Costs } & Mean & 6001 & 6165 & 6001 & 6165 & 6001 & 6165 \\
\hline & $95 \% \mathrm{Cl}$ & 4552 to 7664 & 4657 to 7850 & 4552 to 7664 & 4657 to 7850 & 4552 to 7664 & 4657 to 7850 \\
\hline \multirow[t]{2}{*}{ Incremental costs } & Mean & -164 & & -164 & & -164 & \\
\hline & $95 \% \mathrm{Cl}$ & -2018 to 2502 & & -2018 to 2502 & & -2018 to 2502 & \\
\hline \multirow[t]{2}{*}{ QALYS } & Mean & 1.027 & 0.913 & 0.984 & 0.957 & 1.107 & 1.074 \\
\hline & $95 \% \mathrm{Cl}$ & 0.966 to 1.083 & 0.839 to 0.980 & 0.941 to 1.028 & 0.912 to 1.001 & 1.074 to 1.135 & 1.046 to 1.100 \\
\hline \multirow[t]{2}{*}{ Incremental QALYs } & Mean & 0.114 & & 0.028 & & 0.033 & \\
\hline & $95 \% \mathrm{Cl}$ & 0.026 to $0.206^{*}$ & & -0.031 to 0.090 & & -0.012 to 0.072 & \\
\hline ICER & & $€-1081$ / QALY & & $€ 2049$ / QALY & & $€-6189$ / QALY & \\
\hline \multirow[t]{2}{*}{ INMB at WTP of 0} & Mean & $€ 164$ & & $€-57$ & & $€ 164$ & \\
\hline & $95 \% \mathrm{Cl}$ & $€-2018$ to $€ 2502$ & & $€-2709$ to $€ 2370$ & & $€-2081$ to 2419 & \\
\hline \multirow[t]{2}{*}{ INMB at WTP of $€ 20,000$} & Mean & $€ 2403$ & & $€ 498$ & & $€ 807$ & \\
\hline & $95 \% \mathrm{Cl}$ & $€-893$ to $€ 5454$ & & $€-2514$ to $€ 3269$ & & $€-1898$ to $€ 3249$ & \\
\hline \multirow[t]{2}{*}{ INMB at WTP of $€ 50,000$} & Mean & $€ 5761$ & & $€ 1331$ & & $€ 1770$ & \\
\hline & $95 \% \mathrm{Cl}$ & $€ 386$ to $€ 11,274^{*}$ & & $€-3189$ to $€ 5773$ & & $€-1322$ to $€ 5064$ & \\
\hline
\end{tabular}

NOTE. Unadjusted and adjusted cost-effectiveness analyses used QALYs calculated from EQ-5D utilities, while the QLQ-C30 analysis used QALYs calculated from EORTC QLQ-C30 utilities. Estimates in the adjusted cost-effectiveness analysis were adjusted for baseline EQ-5D utility scores. Confidence intervals were obtained after bootstrapping with 1000 replications

Abbreviations: $b C B T$ blended cognitive behaviour therapy, CAU care as usual, QLQ-C30 European Organisation for Research and Treatment of Cancer Quality of Life Questionnaire C30, ICER incremental cost-effectiveness ratio, INMB incremental net monetary benefit, WTP willingness to pay, QALY quality adjusted life-year *Significant difference between the two conditions $(p<0.05)$

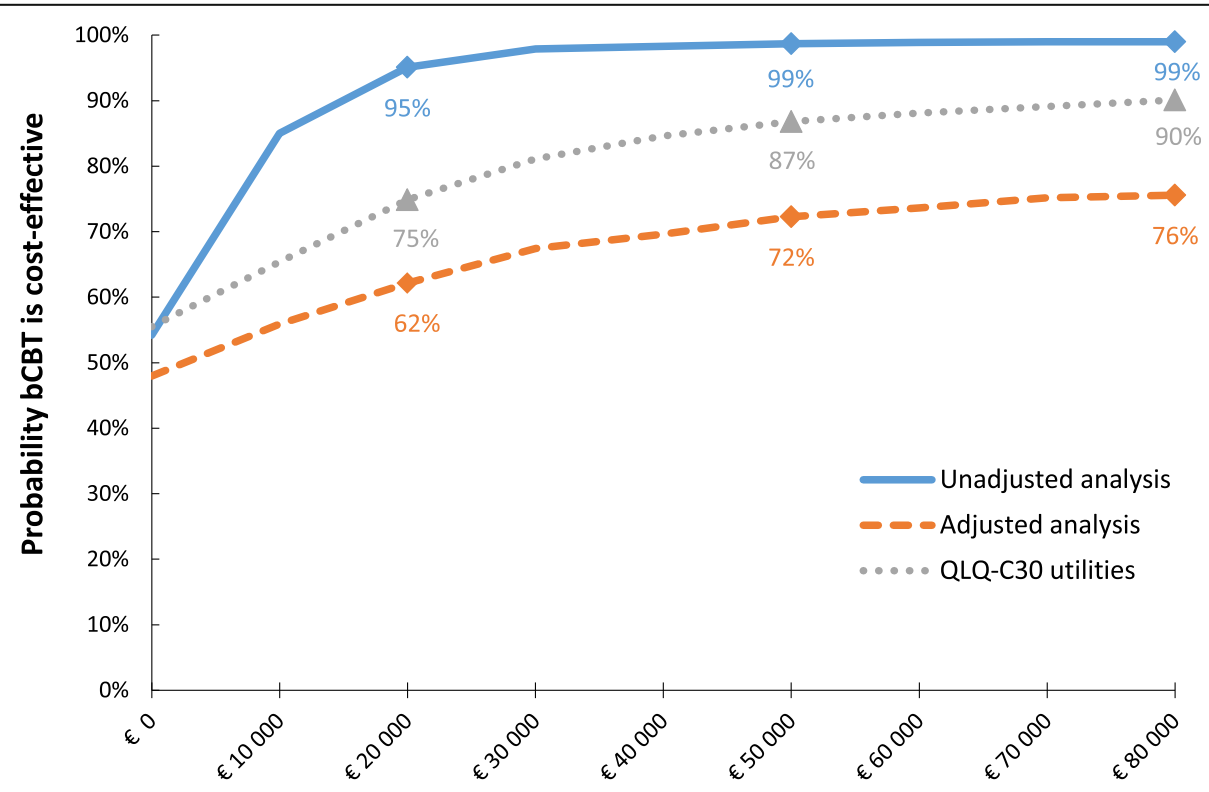

Willingness to pay per QALY

Fig. 2 Cost-effectiveness acceptability curves of the different cost-effectiveness analyses, showing the probability that the intervention is costeffective at different willingness to pay (WTP) thresholds. Abbreviations: bCBT, blended cognitive behaviour therapy; QALY, quality-adjusted life year; QLQ-C30, European Organisation for Research and Treatment of Cancer Quality of Life Questionnaire C30 
participants with recurrence (11\%) was observed during follow-up. Recurrences were self-reported by participants and not systematically investigated in medical records for privacy reasons. More participants in the bCBT than in the CAU condition reported a recurrence, which may have occurred due to higher follow-up adherence of the intervention group. It is noteworthy that a number of participants with recurrence still completed follow-up questionnaires; however, their data after recurrence was excluded from the main analysis since we were only interested in the efficacy and cost-effectiveness of bCBT in disease-free cancer survivors. Although it is possible that the intervention remains suitable for people with recurrent disease, the extent to which bCBT translates to or requires adaptation for patients with advanced disease remains a question for future research.

A further limitation is that this study was not powered to detect differences in cost-effectiveness and therefore these outcomes should be interpreted with caution. Self-reported cost diaries are considered to be feasible and valid for collecting cost data [58], although in the current study it was not possible to distinguish between routine follow-up consultations and consultations due to reassurance-seeking behaviour related to FCR, which may have influenced results. Furthermore, baseline cost data, inability to perform unpaid work, limited job performance while at work and travel costs were not measured.

Baseline differences on EQ-5D utility scores were substantial while all other baseline characteristics were comparable between conditions. These differences are likely to have occurred by chance, as randomisation was performed independently by a computer-generated allocation sequence in the RCT. However, the EQ-5D has been previously reported to be less sensitive to changes in psychological well-being due to dominance of physical health domains [59]. It is possible that the psychological well-being item in the EQ-5D ('anxiety/depression') may not be sensitive to the psychological impact of FCR, as cancer survivors may see FCR as a normal or rational fear and not consider themselves to be anxious or depressed. Indeed, a relatively large proportion of participants in both conditions $(33 \%$ in CAU and $47 \%$ in bCBT) reported no problems with anxiety or depression at baseline, even though all had high FCR at inclusion. Therefore, we additionally used the EORTC QLQ-C30 to calculate QALYs, which may be more sensitive than the EQ-5D in this population. This analysis resulted in higher mean QALY values (as expected) [49], but incremental QALYs were comparable to findings in the adjusted EQ-5D analysis; indicating that those results are robust.

\section{Conclusions}

This study demonstrates that bCBT is statistically and clinically more effective than CAU over the first 15 months post-treatment, and a relatively inexpensive intervention with similar costs and QALYs as CAU. However, results should be replicated in larger samples and in subgroups of the investigated population.

\section{Additional file}

\begin{abstract}
Additional file 1: Appendix 1. Reference prices per healthcare resource category. A list of all healthcare resources measured by the cost diaries in this study and the associated unit cost per resource, which were used to calculate costs per study participant. Appendix 2. Calculation of bCBT intervention programme costs. A description of how bCBT intervention costs were calculated bottom-up; which were subsequently used in the cost-effectiveness analyses for the bCBT group. Appendix 3. Figures of mean CWS scores, mean EQ-5D utility scores and mean EORTC QLQ-C30 utility scores over time by study group. Visual representation of mean CWS scores, EQ-5D utility scores (unadjusted) and EORTC QLQ-C30 utility scores at each follow-up measurement (3, 9, 15 months). Appendix 4. Sensitivity analyses results conducted on the primary CWS outcome. Results of the sensitivity analysis which excluded participants who reported a recurrence and the per protocol sensitivity analysis, which only included participants who completed the intervention and who had full follow-up FCR data. Appendix 5. Reliable improvement, clinically significant change, clinically significant improvement and self-rated improvement in FCR severity. Results of analyses which investigated the clinical relevance of FCR improvement at 9 months and 15 months follow-up by treatment group for complete cases. Results of clinical relevancy analyses at 3 months follow-up are published elsewhere [15]. Appendix 6. Costs per assessment per resource category. Table of mean costs measured per resource category per follow-up assessment per study group. Costs per resource category are listed only for complete cases, since missing cost data were imputed at the aggregated (total cost) level. (DOCX $176 \mathrm{~kb})$
\end{abstract}

\section{Abbreviations}

BC: curatively-treated breast cancer survivor; bCBT: blended cognitive behaviour therapy; CAU: Care as usual; CEAC: Cost-effectiveness acceptability curve; CRC: Curatively-treated colorectal cancer survivor; CWS: Cancer worry scale; FCR: Fear of cancer recurrence; ICER: Incremental cost-effectiveness ratio; INMB: Incremental net monetary benefit; MI: Multiple imputation; mITT: Modified intention-to-treat; PC: curatively-treated prostate cancer survivor; QALY: Quality-adjusted life-years; RCT: Randomised controlled trial; WTP: Willingness to pay

\section{Acknowledgements}

We are grateful to the patients for their participation in this trial and to the oncologists, radiotherapists, urologists and nurse specialists of the Radboud University Medical Center, Nijmegen; Canisius-Wilhelmina Hospital, Nijmegen; Maasziekenhuis Pantein, Boxmeer; Hospital Bernhoven, Uden/Neghel and Hospital Gelderse Vallei Ede for their efforts in referring patients to the trial. We would like to thank Dr. P. Servaes and D. Marcelissen for carrying out the intervention.

\section{Funding}

This research is funded by the Dutch Cancer Society (Grant number KUN 2012-5545). This funding source had no role in the design of this study, execution, analyses, interpretation of data or decision to submit the results.

\section{Availability of data and materials}

All data generated or analysed during this study are available from the corresponding author on reasonable request. 


\section{Authors' contributions}

Conception and design: JP, AS. Acquisition of data: MW. Analysis and interpretation of data: $R B$, LR. Drafting of manuscript: RB, BT, LR, MW. Critical revision: RB, BT, JP, LR, MW, WK, AS. All authors read and approved the final manuscript.

\section{Ethics approval and consent to participate}

The RCT was approved by an ethical board (CMO Arnhem-Nijmegen). Written informed consent was obtained from all eligible participants.

\section{Consent for publication}

Not applicable.

\section{Competing interests}

The authors declare that they have no competing interests.

\section{Publisher's Note}

Springer Nature remains neutral with regard to jurisdictional claims in published maps and institutional affiliations.

\begin{abstract}
Author details
'Department of Medical Psychology, Radboud university medical center, Radboud Institute for Health Sciences, Nijmegen, The Netherlands. ${ }^{2}$ MedValue, PO Box 9101, 6500, HB, Nijmegen, The Netherlands. ${ }^{3}$ Department for Health Evidence, Radboud university medical center, Radboud Institute for Health Sciences, Nijmegen, The Netherlands. ${ }^{4}$ Department of Psychiatry, Radboud university medical center, Radboud Institute for Health Sciences, Nijmegen, The Netherlands. ${ }^{5}$ Maxima Medical Center, Department of Medical Psychology, Eindhoven/Veldhoven, The Netherlands.
\end{abstract}

\section{Received: 11 November 2018 Accepted: 15 April 2019}

\section{Published online: 16 May 2019}

\section{References}

1. Vickberg SM. The concerns about recurrence scale (CARS): a systematic measure of women's fears about the possibility of breast cancer recurrence. Ann Behav Med. 2003:25(1):16-24.

2. Lebel S, Ozakinci G, Humphris G, Mutsaers B, Thewes B, Prins J, et al. From normal response to clinical problem: definition and clinical features of fear of cancer recurrence. Support Care Cancer. 2016;24(8):3265-8. https://doi. org/10.1007/s00520-016-3272-5.

3. Simard S, Thewes B, Humphris G, Dixon M, Hayden C, Mireskandari S, et al. Fear of cancer recurrence in adult cancer survivors: a systematic review of quantitative studies. J Cancer Surviv. 2013;7(3):300-22. https://doi.org/10. 1007/s11764-013-0272-z.

4. Tewari A, Chagpar AB. Worry about breast cancer recurrence: a populationbased analysis. Am Surg. 2014;80(7):640-5.

5. Koch $L$, Jansen $L$, Brenner $H$, Arndt $V$. Fear of recurrence and disease progression in long-term ( $>/=5$ years) cancer survivors--a systematic review of quantitative studies. Psycho-oncology. 2013;22(1):1-11. https://doi.org/10. 1002/pon.3022.

6. Lebel S, Tomei C, Feldstain A, Beattie S, McCallum M. Does fear of cancer recurrence predict cancer survivors' health care use? Support Care Cancer. 2013;21(3):901-6. https://doi.org/10.1007/s00520-012-1685-3.

7. Lee-Jones C, Humphris G, Dixon R, Hatcher MB. Fear of cancer recurrence--a literature review and proposed cognitive formulation to explain exacerbation of recurrence fears. Psycho-oncology. 1997;6(2):95-105. https:// doi.org/10.1002/(sici)1099-1611(199706)6:2<95:.aid-pon250>3.0.co;2-b.

8. Sarkar S, Sautier L, Schilling G, Bokemeyer C, Koch U, Mehnert A. Anxiety and fear of cancer recurrence and its association with supportive care needs and health-care service utilization in cancer patients. I Cancer Surviv. 2015;9(4):567-75. https://doi.org/10.1007/s11764-015-0434-2.

9. Custers JA, Gielissen MF, Janssen SH, de Wilt JH, Prins JB. Fear of cancer recurrence in colorectal cancer survivors. Support Care Cancer. 2016;24(2): 555-62. https://doi.org/10.1007/s00520-015-2808-4.

10. Custers JA, van den Berg SW, van Laarhoven HW, Bleiker EM, Gielissen MF, Prins JB. The Cancer worry scale: detecting fear of recurrence in breast cancer survivors. Cancer Nurs. 2014;37(1):E44-50. https://doi.org/10.1097/ NCC.0b013e3182813a17.
11. van de Wal M, van Oort I, Schouten J, Thewes B, Gielissen M, Prins J. Fear of cancer recurrence in prostate cancer survivors. Acta oncologica (Stockholm, Sweden). 2016;55(7):821-7. https://doi.org/10.3109/0284186x.2016.1150607.

12. van de Wal M, van de Poll-Franse L, Prins J, Gielissen M. Does fear of cancer recurrence differ between cancer types? A study from the population-based PROFILES registry. Psycho-oncology. 2016;25(7):772-8. https://doi.org/10. 1002/pon.4002.

13. Dieng M, Butow PN, Costa DS, Morton RL, Menzies SW, Mireskandari S, et al. Psychoeducational intervention to reduce fear of Cancer recurrence in people at high risk of developing another primary melanoma: results of a randomized controlled trial. J Clin Oncol. 2016;34(36):4405-14. https://doi. org/10.1200/jco.2016.68.2278.

14. van de Wal M, Thewes B, Gielissen M, Speckens A, Prins J. Efficacy of blended cognitive behavior therapy for high fear of recurrence in breast, prostate, and colorectal Cancer survivors: the SWORD study, a randomized controlled trial. J Clin Oncol. 2017;35(19):2173-83. https://doi.org/10.1200/ jco.2016.70.5301.

15. Humphris G, Rogers $S$. AFTER and beyond: cancer recurrence fears and a test of an intervention in oral and oropharyngeal patients. Soc Sci Dent. 2012;2:29-38.

16. Butow PN, Turner J, Gilchrist J, Sharpe L, Smith AB, Fardell JE, et al. Randomized Trial of ConquerFear: A Novel, Theoretically Based Psychosocial Intervention for Fear of Cancer Recurrence. J Clin Oncol. 2017: Jco2017731257. https://doi.org/10.1200/jco.2017.73.1257.

17. van den Berg SW, Gielissen MF, Custers JA, van der Graaf WT, Ottevanger PB, Prins JB. BREATH: web-based self-Management for Psychological Adjustment after Primary Breast Cancer--Results of a multicenter randomized controlled trial. J Clin Oncol. 2015;33(25):2763-71. https://doi. org/10.1200/jco.2013.54.9386.

18. Owen JE, Klapow JC, Roth DL, Shuster JL Jr, Bellis J, Meredith R, et al. Randomized pilot of a self-guided internet coping group for women with early-stage breast cancer. Ann Behav Med. 2005;30(1):54-64. https://doi.org/ 10.1207/s15324796abm3001_7.

19. Leykin Y, Thekdi SM, Shumay DM, Munoz RF, Riba M, Dunn LB. Internet interventions for improving psychological well-being in psycho-oncology: review and recommendations. Psycho-oncology. 2012;21(9):1016-25. https://doi.org/10.1002/pon.1993.

20. Munoz RF. Using evidence-based internet interventions to reduce health disparities worldwide. J Med Internet Res. 2010;12(5):e60. https://doi.org/10. 2196/jmir.1463.

21. Donker T, Blankers M, Hedman E, Ljotsson B, Petrie K, Christensen H. Economic evaluations of internet interventions for mental health: a systematic review. Psychol Med. 2015;45(16):3357-76. https://doi.org/10. 1017/s0033291715001427.

22. Andrews G, Cuijpers P, Craske MG, McEvoy P, Titov N. Computer therapy for the anxiety and depressive disorders is effective, acceptable and practical health care: a meta-analysis. PLoS One. 2010;5(10):e13196. https://doi.org/10. 1371/journal.pone.0013196.

23. Spek V, Cuijpers P, Nyklicek I, Riper H, Keyzer J, Pop V. Internet-based cognitive behaviour therapy for symptoms of depression and anxiety: a meta-analysis. Psychol Med. 2007:37(3):319-28. https://doi.org/10.1017/ s0033291706008944

24. Haug T, Nordgreen T, Ost LG, Havik OE. Self-help treatment of anxiety disorders: a meta-analysis and meta-regression of effects and potential moderators. Clin Psychol Rev. 2012;32(5):425-45. https://doi.org/10.1016/j. cpr.2012.04.002

25. Carlbring P, Nilsson-Ihrfelt E, Waara J, Kollenstam C, Buhrman M, Kaldo V, et al. Treatment of panic disorder: live therapy vs. self-help via the internet. Behav Res Ther. 2005;43(10):1321-33. https://doi.org/10.1016/j.brat.2004.10. 002.

26. Wilhelmsen M, Lillevoll K, Risor MB, Hoifodt R, Johansen ML, Waterloo K, et al. Motivation to persist with internet-based cognitive behavioural treatment using blended care: a qualitative study. BMC psychiatry. 2013;13: 296. https://doi.org/10.1186/1471-244x-13-296.

27. van de Wal MA, Gielissen MF, Servaes P, Knoop H, Speckens AE, Prins JB. Study protocol of the SWORD-study: a randomised controlled trial comparing combined online and face-to-face cognitive behaviour therapy versus treatment as usual in managing fear of cancer recurrence. BMC psychology. 2015;3(1):12. https://doi.org/10.1186/s40359-015-0068-1.

28. Sabariego C, Brach M, Herschbach P, Berg P, Stucki G. Cost-effectiveness of cognitive-behavioral group therapy for dysfunctional fear of progression in 
cancer patients. Eur J Health Econ. 2011;12(5):489-97. https://doi.org/10. 1007/s10198-010-0266-y.

29. Richtlijn voor het uitvoeren van economische evaluaties in de gezondheidszorg - Guideline for conducting economic evaluations in healthcare. Zorginstituut Nederland 2016.

30. Simard S, Savard J. Fear of Cancer recurrence inventory: development and initial validation of a multidimensional measure of fear of cancer recurrence. Support Care Cancer. 2009;17(3):241-51. https://doi.org/10.1007/s00520-0080444-y.

31. Horowitz M, Wilner N, Alvarez W. Impact of event scale: a measure of subjective stress. Psychosom Med. 1979;41(3):209-18.

32. van der Ploeg E, Mooren TT, Kleber RJ, van der Velden PG, Brom D. Construct validation of the Dutch version of the impact of event scale Psychol Assess. 2004;16(1):16-26. https://doi.org/10.1037/1040-3590.16.1.16.

33. Zigmond AS, Snaith RP. The hospital anxiety and depression scale. Acta Psychiatr Scand. 1983;67(6):361-70.

34. Servaes $P$, Prins J, Verhagen $S$, Bleijenberg G. Fatigue after breast cancer and in chronic fatigue syndrome: similarities and differences. J Psychosom Res. 2002;52(6):453-9.

35. Aaronson NK, Ahmedzai S, Bergman B, Bullinger M, Cull A, Duez NJ, et al. The European Organization for Research and Treatment of Cancer QLQ-C30: a quality-of-life instrument for use in international clinical trials in oncology. J Natl Cancer Inst. 1993;85(5):365-76.

36. Group TE. EuroQol--a new facility for the measurement of health-related quality of life. Health policy (Amsterdam, Netherlands). 1990;16(3):199-208,

37. Lamers LM, Stalmeier PF, McDonnell J, Krabbe PF, van Busschbach JJ. Measuring the quality of life in economic evaluations: the Dutch EQ-5D tariff. Ned Tijdschr Geneeskd. 2005;149(28):1574-8.

38. Koopmanschap MA, Rutten FF, van Ineveld BM, van Roijen L. The friction cost method for measuring indirect costs of disease. J Health Econ. 1995; 14(2):171-89.

39. Drummond MSM, Torrance G, O'Brien B, Stoddart G. Methods for the Economic Evaluation of Health Care Programmes. 3rd ed. Oxford: UK: Oxford University Press; 2005.

40. Bergmo TS. How to Measure Costs and Benefits of eHealth Interventions: An Overview of Methods and Frameworks. J Med Internet Res. 2015;17(11). https://doi.org/10.2196/jmir.4521.

41. Jacobson NS, Truax P. Clinical significance: a statistical approach to defining meaningful change in psychotherapy research. J Consult Clin Psychol. 1991; 59(1):12-9.

42. Faria R, Gomes M, Epstein D, White IR. A guide to handling missing data in cost-effectiveness analysis conducted within randomised controlled trials. PharmacoEconomics. 2014;32(12):1157-70. https://doi.org/10.1007/s40273014-0193-3.

43. Simons $\mathrm{CL}$, Rivero-Arias $\mathrm{O}$, Yu LM, Simon J. Multiple imputation to deal with missing EQ-5D-3L data: should we impute individual domains or the actual index? Qual Life Res. 2015;24(4):805-15. https://doi.org/10.1007/s11136-0140837-y.

44. Rubin DB, Schenker N. Multiple imputation in health-care databases: an overview and some applications. Stat Med. 1991;10(4):585-98.

45. Thompson SG, Barber JA. How should cost data in pragmatic randomised trials be analysed? BMJ (Clinical research ed). 2000;320(7243):1197-200.

46. Briggs $\mathrm{AH}$, Wonderling DE, Mooney CZ. Pulling cost-effectiveness analysis up by its bootstraps: a non-parametric approach to confidence interval estimation. Health Econ. 1997;6(4):327-40.

47. Hoch JS, Dewa CS. Advantages of the net benefit regression framework for economic evaluations of interventions in the workplace: a case study of the cost-effectiveness of a collaborative mental health care program for people receiving short-term disability benefits for psychiatric disorders. J Occup Environ Med. 2014;56(4):441-5. https://doi.org/10.1097/jom. 0000000000000130.

48. Hoch JS, Rockx MA, Krahn AD. Using the net benefit regression framework to construct cost-effectiveness acceptability curves: an example using data from a trial of external loop recorders versus Holter monitoring for ambulatory monitoring of "community acquired" syncope. BMC Health Serv Res. 2006;6:68. https://doi.org/10.1186/1472-6963-6-68.

49. Versteegh MM, Leunis A, Uyl-de Groot CA, Stolk EA. Condition-specific preference-based measures: benefit or burden? Value Health. 2012;15(3): 504-13. https://doi.org/10.1016/j.jval.2011.12.003.

50. Symposium Abstracts. Psycho-oncology. 2018;27(S3):8-55. doi:https://doi. org/10.1002/pon.4877.
51. Savard J, Ivers $\mathrm{H}$. The evolution of fear of cancer recurrence during the cancer care trajectory and its relationship with cancer characteristics. J Psychosom Res. 2013;74(4):354-60. https://doi.org/10.1016/j.jpsychores.2012. 12.013.

52. Crist JV, Grunfeld EA. Factors reported to influence fear of recurrence in cancer patients: a systematic review. Psycho-oncology. 2013;22(5):978-86. https://doi.org/10.1002/pon.3114

53. Kosteneffectiviteit in de praktijk. Zorginstituut Nederland 2015.

54. Tate DF, Finkelstein EA, Khavjou O, Gustafson A. Cost effectiveness of internet interventions: review and recommendations. Ann Behav Med. 2009; 38(1):40-5. https://doi.org/10.1007/s12160-009-9131-6.

55. van Helmondt SJ, van der Lee ML, de Vries J. Study protocol of the CARESTtrial: a randomised controlled trial on the (cost-) effectiveness of a CBTbased online self-help training for fear of cancer recurrence in women with curatively treated breast cancer. BMC Cancer. 2016;16:527. https://doi.org/10. 1186/s12885-016-2562-0

56. Dieng M, Cust AE, Kasparian NA, Butow P, Costa DS, Menzies SW, et al. Protocol for a within-trial economic evaluation of a psychoeducational intervention tailored to people at high risk of developing a second or subsequent melanoma. BMJ Open. 2016;6(10):e012153. https://doi.org/10. 1136/bmjopen-2016-012153.

57. Butow PN, Bell ML, Smith AB, Fardell JE, Thewes B, Turner J, et al. Conquer fear: protocol of a randomised controlled trial of a psychological intervention to reduce fear of cancer recurrence. BMC Cancer. 2013;13:201. https://doi.org/10.1186/1471-2407-13-201.

58. Goossens ME, Rutten-van Molken MP, Vlaeyen JW, van der Linden SM. The cost diary: a method to measure direct and indirect costs in costeffectiveness research. J Clin Epidemiol. 2000;53(7):688-95.

59. van de Willige G, Wiersma D, Nienhuis FJ, Jenner JA. Changes in quality of life in chronic psychiatric patients: a comparison between EuroQol (EQ-5D) and WHOQoL. Qual Life Res. 2005;14(2):441-51.

\section{Ready to submit your research? Choose BMC and benefit from:}

- fast, convenient online submission

- thorough peer review by experienced researchers in your field

- rapid publication on acceptance

- support for research data, including large and complex data types

- gold Open Access which fosters wider collaboration and increased citations

- maximum visibility for your research: over $100 \mathrm{M}$ website views per year

At BMC, research is always in progress.

Learn more biomedcentral.com/submissions 